\title{
New global a priori estimates for the third-grade fluid equations
}

\author{
Mark Steinhauer ${ }^{1}$, José Miguel Urbano ${ }^{2, *, \dagger}$ and Juha Videman ${ }^{3, \ddagger}$ \\ ${ }^{1}$ Mathematisches Seminar, Universität Bonn, Nussallee, 15, D-53115 Bonn, Germany \\ ${ }^{2}$ Departamento de Matemática, Universidade de Coimbra, 3001-454 Coimbra, Portugal \\ ${ }^{3}$ Departamento de Matemática, Instituto Superior Técnico, Av. Rovisco Pais, 1049-001 Lisboa, Portugal
}

\section{Communicated by P. Colli}

\begin{abstract}
SUMMARY
This note bridges the gap between the existence and regularity classes for the third-grade RivlinEricksen fluid equations. We obtain a new global a priori estimate, which conveys the precise regularity conditions that lead to the existence of a global in time regular solution. Copyright (C) 2006 John Wiley \& Sons, Ltd.
\end{abstract}

KEY WORDS: third-grade fluid equations; global a priori estimates; regularity class

\section{INTRODUCTION}

Many models governing the motion of incompressible viscoelastic fluids are best described as systems of non-linear parabolic-hyperbolic PDE's. Typically, existence results for this type of systems can only be obtained locally in time, or else globally, while assuming that the given data are sufficiently small, see, e.g. References [1-9]. For some models, the situation is better in a two-dimensional setting and solvability can be proved globally in time for any sufficiently regular set of data, cf. References [4,10]. Quite recently it was shown, without any smallness assumptions on the data, that the equations of third-grade Rivlin-Ericksen fluids admit global solutions if the initial fluid velocity belongs to $H^{2}\left(\mathbb{R}^{n}\right), n=2,3$, see Reference [10]. In the 2-D case, this regularity is enough to show uniqueness but in the 3-D case there is a gap between the existence and the uniqueness classes.

\footnotetext{
${ }^{*}$ Correspondence to: José Miguel Urbano, Departamento de Matemática, Universidade de Coimbra, 3001-454 Coimbra, Portugal.

†E-mail: jmurb@mat.uc.pt

‡E-mail: videman@math.ist.utl.pt

Contract/grant sponsor: ICCTI/DAAD

Contract/grant sponsor: CMUC/FCT

Contract/grant sponsor: CEMAT/FCT
}

Copyright (c) 2006 John Wiley \& Sons, Ltd.

Received 27 September 2005 
In this article, we will study the regularity of a global in time solution of the thirdgrade fluid equations in 3-D. Our analysis is based on a new global a priori estimate which allows for the study of the precise regularity conditions that lead to the existence of a global regular solution. Consequently, we obtain a regularity class, different from the existence class, but within which the uniqueness is also valid. Let us stress that although the existence of more regular (even classical) solutions for these equations has been studied previously, cf. References [2,9], these results, which are all only true under restrictive smallness (and regularity) conditions on the data or on the material constants, were obtained directly within the regularity and uniqueness classes. Hence, they can only be results 'in the small'.

Finally, it is interesting to note that the term which makes it possible to show global existence in 3-D for third-grade fluids (and not for the second-grade fluid equations in which this term is missing) is the same which Ladyzhenskaya added to the Navier-Stokes equations in order to prove existence of a unique global in time solution, cf. Reference [11]. In our model, however, uniqueness and regularity demand an extra hypothesis due to the presence of higher-order non-linear terms.

The paper is organized as follows: in Section 2 we present the model and introduce some notation; Section 3 gathers the basic a priori estimates for the solution of the problem; Section 4 contains a uniqueness result; in the main Section 5 we bridge the gap between the existence and regularity classes by establishing a new global a priori estimate for the third-order spatial derivatives using an additional regularity assumption.

\section{THE EQUATIONS}

In an incompressible Rivlin-Ericksen fluid of grade three the extra-stress tensor is given by (see Reference [12])

$$
\mathbf{T}_{\mathrm{E}}=\eta \mathbf{A}_{1}(\mathbf{v})+\alpha_{1} \mathbf{A}_{2}(\mathbf{v})+\alpha_{2} \mathbf{A}_{1}^{2}(\mathbf{v})+\beta\left(\operatorname{tr} \mathbf{A}_{1}^{2}(\mathbf{v})\right) \mathbf{A}_{1}(\mathbf{v})
$$

where $\mathbf{v}$ is the fluid velocity, $\mathbf{A}_{1}(\mathbf{v})$ and $\mathbf{A}_{2}(\mathbf{v})$ denote the first two Rivlin-Ericksen tensors

$$
\begin{aligned}
& \mathbf{A}_{1}(\mathbf{v})=\nabla \mathbf{v}+(\nabla \mathbf{v})^{\mathrm{T}} \\
& \mathbf{A}_{2}(\mathbf{v})=\left(\frac{\partial}{\partial t}+\mathbf{v} \cdot \nabla\right) \mathbf{A}_{1}(\mathbf{v})+\mathbf{A}_{1}(\mathbf{v}) \nabla \mathbf{v}+(\nabla \mathbf{v})^{\mathrm{T}} \mathbf{A}_{1}(\mathbf{v})
\end{aligned}
$$

and $\eta, \alpha_{1}, \alpha_{2}$ and $\beta$ stand for material constants. In fact, the constitutive relation (1) is a degenerate form of a more general Rivlin-Ericksen fluid of grade three defined by

$$
\mathbf{T}_{\mathrm{E}}=\eta \mathbf{A}_{1}+\alpha_{1} \mathbf{A}_{2}+\alpha_{2} \mathbf{A}_{1}^{2}+\beta_{1} \mathbf{A}_{3}+\beta_{2}\left(\mathbf{A}_{1} \mathbf{A}_{2}+\mathbf{A}_{2} \mathbf{A}_{1}\right)+\beta_{3}\left(\operatorname{tr} \mathbf{A}_{1}^{2}\right) \mathbf{A}_{1}
$$

and obtained by assuming, in view of thermodynamics, that $\beta_{1}=\beta_{2}=0$ (see Reference [13]).

A third-grade fluid is compatible with thermodynamics if the material constants in (1) satisfy the conditions (cf. Reference [13]):

$$
\eta \geqslant 0, \quad \alpha_{1} \geqslant 0, \quad \beta \geqslant 0, \quad\left|\alpha_{1}+\alpha_{2}\right| \leqslant \sqrt{24 \eta \beta}
$$


The constitutive law (1) includes as special cases the fluids of second-grade $(\beta=0)$, and the Newtonian fluids $\left(\beta=\alpha_{1}=\alpha_{2}=0\right)$.

The constitutive relation (1), together with the equations of motion, leads to the following system of equations - for $\mathbf{v}$ and the hydrostatic pressure $p$ (redefined after division by the constant density) - that governs the motion of an incompressible viscoelastic Rivlin-Ericksen fluid of grade three:

$$
\begin{cases}\frac{\partial}{\partial t}\left(\mathbf{v}-\alpha_{1} \Delta \mathbf{v}\right)-v \Delta \mathbf{v}-\beta \nabla \cdot\left(|\mathbf{A}(\mathbf{v})|^{2} \mathbf{A}(\mathbf{v})\right)+\nabla p & \\ =-\mathbf{v} \cdot \nabla\left(\mathbf{v}-\alpha_{1} \Delta \mathbf{v}\right)+\nabla \cdot \mathbf{N}(\mathbf{v})+\mathbf{f} & \text { in } \mathbb{R}^{3} \times(0, T) \\ \nabla \cdot \mathbf{v}=0 & \text { in } \mathbb{R}^{3} \times(0, T) \\ \mathbf{v}(x, 0)=\mathbf{v}_{0}(x) & x \in \mathbb{R}^{3}\end{cases}
$$

Here we have set $\mathbf{A}(\mathbf{v})=\mathbf{A}_{1}(\mathbf{v})$. Moreover, all the material constants are divided by the constant density $\rho(v=\eta / \rho$ denotes the kinematic viscosity coefficient $)$, the vector field $\mathbf{f}$ stands for external body forces, and

$$
\mathbf{N}(\mathbf{v})=\alpha_{1}(\nabla \mathbf{v})^{\mathrm{T}} \mathbf{A}(\mathbf{v})+\left(\alpha_{1}+\alpha_{2}\right) \mathbf{A}^{2}(\mathbf{v})
$$

\section{BASIC A PRIORI ESTIMATES}

Our main result is based on a new, global a priori estimate for the third-order spatial derivatives of the solution. Hence, for the sake of completeness, we gather in this section a few basic a priori estimates for the lower-order derivatives that will be useful in the sequel and briefly recall how they can be derived. We will assume throughout the paper that $v, \alpha_{1}$ and $\beta$ are strictly positive. Let us start by recalling the existence result proven in Reference [10].

\section{Theorem 3.1}

Assume that $\mathbf{f} \in L_{\mathrm{loc}}^{\infty}\left([0, \infty) ; L^{2}\left(\mathbb{R}^{3}\right)\right)$ and that $\mathbf{v}_{0} \in H^{2}\left(\mathbb{R}^{3}\right)$, with $\nabla \cdot \mathbf{v}_{0}=0$. There exists a solution $\mathbf{v} \in C_{\mathrm{w}}\left([0, T) ; H^{2}\left(\mathbb{R}^{3}\right)\right)$, which is global in time (i.e. the solution exists for all $T>0$ ), satisfying Equations (4) in the sense of distributions.

The result follows from an a priori estimate for the $L^{2}\left(\mathbb{R}^{3}\right)$-norm of $\mathbf{v}-\alpha_{1} \Delta \mathbf{v}$ and from a subsequent application of the Galerkin method.

We next describe how to obtain the basic a priori estimates. Multiplying $(4)_{1}$ with $\mathbf{v}$, integrating over $\mathbb{R}^{3}$, performing several integrations by parts, and using the fact that $\nabla \cdot \mathbf{v}=0$, one obtains

$$
\begin{aligned}
& \frac{1}{2} \frac{\mathrm{d}}{\mathrm{d} t}\left(\int_{\mathbb{R}^{3}}|\mathbf{v}|^{2} \mathrm{~d} x+\alpha_{1} \int_{\mathbb{R}^{3}}|\nabla \mathbf{v}|^{2} \mathrm{~d} x\right)+v \int_{\mathbb{R}^{3}}|\nabla \mathbf{v}|^{2} \mathrm{~d} x+\frac{\beta}{2} \int_{\mathbb{R}^{3}}|\mathbf{A}(\mathbf{v})|^{4} \mathrm{~d} x \\
& \quad=-\frac{\alpha_{1}+\alpha_{2}}{2} \int_{\mathbb{R}^{3}} \mathbf{A}^{2}(\mathbf{v}): \mathbf{A}(\mathbf{v}) \mathrm{d} x+\int_{\mathbb{R}^{3}} \mathbf{f} \cdot \mathbf{v} \mathrm{d} x
\end{aligned}
$$


where $\mathbf{A}: \mathbf{B}=A_{i j} B_{i j}$ denotes the usual double scalar product between two second-order tensors. Using Hölder's and Young's inequalities, we get

$$
\begin{aligned}
& \frac{\mathrm{d}}{\mathrm{d} t}\left(\int_{\mathbb{R}^{3}}|\mathbf{v}|^{2} \mathrm{~d} x+\alpha_{1} \int_{\mathbb{R}^{3}}|\nabla \mathbf{v}|^{2} \mathrm{~d} x\right)+2 v \int_{\mathbb{R}^{3}}|\nabla \mathbf{v}|^{2} \mathrm{~d} x+\frac{\beta}{2} \int_{\mathbb{R}^{3}}|\mathbf{A}(\mathbf{v})|^{4} \mathrm{~d} x \\
& \quad \leqslant \frac{\left|\alpha_{1}+\alpha_{2}\right|^{2}}{2 \alpha_{1} \beta}\left(\alpha_{1} \int_{\mathbb{R}^{3}}|\nabla \mathbf{v}|^{2} \mathrm{~d} x+\int_{\mathbb{R}^{3}}|\mathbf{v}|^{2} \mathrm{~d} x\right)+\frac{2 \alpha_{1} \beta}{\left|\alpha_{1}+\alpha_{2}\right|^{2}} \int_{\mathbb{R}^{3}}|\mathbf{f}|^{2} \mathrm{~d} x
\end{aligned}
$$

and, hence, Gronwall's inequality yields the first a priori estimate

$$
\begin{aligned}
& \text { ess } \sup _{0 \leqslant t \leqslant T}\left(\|\mathbf{v}\|_{0,2}^{2}+\alpha_{1}\|\nabla \mathbf{v}\|_{0,2}^{2}\right)+2 v \int_{0}^{T}\|\nabla \mathbf{v}\|_{0,2}^{2} \mathrm{~d} t+\frac{\beta}{2} \int_{0}^{\mathrm{T}}\|\mathbf{A}(\mathbf{v})\|_{0,4}^{4} \mathrm{~d} t \\
& \quad \leqslant \exp \left\{\frac{\left|\alpha_{1}+\alpha_{2}\right|^{2}}{\beta \alpha_{1}} T\right\}\left\{\left\|\mathbf{v}_{0}\right\|_{0,2}^{2}+\alpha_{1}\left\|\nabla \mathbf{v}_{0}\right\|_{0,2}^{2}+\frac{2 \alpha_{1} \beta}{\left|\alpha_{1}+\alpha_{2}\right|^{2}} \int_{0}^{T}\|\mathbf{f}\|_{0,2}^{2} \mathrm{~d} t\right\}
\end{aligned}
$$

Next, let us (formally) multiply Equation $(4)_{1}$ by $-\Delta \mathbf{v}$, integrate over $\mathbb{R}^{3}$, and again integrate by parts. This results in

$$
\begin{aligned}
& \frac{1}{2} \frac{\mathrm{d}}{\mathrm{d} t}\left(\int_{\mathbb{R}^{3}}|\nabla \mathbf{v}|^{2} \mathrm{~d} x+\alpha_{1} \int_{\mathbb{R}^{3}}\left|\nabla^{2} \mathbf{v}\right|^{2} \mathrm{~d} x\right)+v \int_{\mathbb{R}^{3}}\left|\nabla^{2} \mathbf{v}\right|^{2} \mathrm{~d} x \\
& \quad+\frac{\beta}{2} \int_{\mathbb{R}^{3}}|\mathbf{A}(\mathbf{v})|^{2}|\nabla \mathbf{A}(\mathbf{v})|^{2} \mathrm{~d} x+\beta \sum_{j} \int_{\mathbb{R}^{3}}\left(\mathbf{A}(\mathbf{v}): \partial_{j} \mathbf{A}(\mathbf{v})\right)^{2} \mathrm{~d} x \\
& \leqslant\left|\alpha_{1}+\alpha_{2}\right| \int_{\mathbb{R}^{3}}|\nabla \mathbf{A}(\mathbf{v})|^{2}|\mathbf{A}(\mathbf{v})| \mathrm{d} x+\frac{\alpha_{1}}{2} \int_{\mathbb{R}^{3}}|\mathbf{A}(\mathbf{v})||\nabla \mathbf{A}(\mathbf{v})|^{2} \mathrm{~d} x \\
& \quad+\int_{\mathbb{R}^{3}}|\mathbf{v}||\mathbf{A}(\mathbf{v})||\nabla \mathbf{A}(\mathbf{v})| \mathrm{d} x+|(\mathbf{f}, \Delta \mathbf{v})|
\end{aligned}
$$

Using Hölder's and Young's inequalities, one can absorb part of the terms on the right-hand side of (7) to the other side. This leads to

$$
\begin{aligned}
& \frac{\mathrm{d}}{\mathrm{d} t}\left(\int_{\mathbb{R}^{3}}|\nabla \mathbf{v}|^{2} \mathrm{~d} x+\alpha_{1} \int_{\mathbb{R}^{3}}\left|\nabla^{2} \mathbf{v}\right|^{2} \mathrm{~d} x\right)+v \int_{\mathbb{R}^{3}}\left|\nabla^{2} \mathbf{v}\right|^{2} \mathrm{~d} x \\
& \quad+\frac{\beta}{4} \int_{\mathbb{R}^{3}}|\mathbf{A}(\mathbf{v})|^{2}|\nabla \mathbf{A}(\mathbf{v})|^{2} \mathrm{~d} x+2 \beta \sum_{j} \int_{\mathbb{R}^{3}}\left(\mathbf{A}(\mathbf{v}): \partial_{j} \mathbf{A}(\mathbf{v})\right)^{2} \mathrm{~d} x \\
& \quad \leqslant \frac{4}{\beta}\left(4\left|\alpha_{1}+\alpha_{2}\right|^{2}+\alpha_{1}^{2}\right) \int_{\mathbb{R}^{3}}\left|\nabla^{2} \mathbf{v}\right|^{2} \mathrm{~d} x+\frac{4}{\beta} \int_{\mathbb{R}^{3}}|\mathbf{v}|^{2} \mathrm{~d} x+\frac{1}{v} \int_{\mathbb{R}^{3}}|\mathbf{f}|^{2} \mathrm{~d} x
\end{aligned}
$$


and one obtains, from Gronwall's inequality,

$$
\begin{aligned}
& \text { ess } \sup _{0 \leqslant t \leqslant T}\left(\|\nabla \mathbf{v}\|_{0,2}^{2}+\alpha_{1}\left\|\nabla^{2} \mathbf{v}\right\|_{0,2}^{2}\right)+v \int_{0}^{T}\left\|\nabla^{2} \mathbf{v}\right\|_{0,2}^{2} \mathrm{~d} t+\frac{\beta}{4} \int_{0}^{T} \int_{\mathbb{R}^{3}}|\mathbf{A}(\mathbf{v})|^{2}|\nabla \mathbf{A}(\mathbf{v})|^{2} \mathrm{~d} x \mathrm{~d} t \\
& \quad \leqslant \exp \left\{\left(\frac{384 v}{\alpha_{1}}+\frac{4 \alpha_{1}}{\beta}\right) T\right\} \cdot\left(\left\|\nabla \mathbf{v}_{0}\right\|_{0,2}^{2}+\alpha_{1}\left\|\nabla^{2} \mathbf{v}_{0}\right\|_{0,2}^{2}+\frac{4}{\beta} \int_{0}^{T}\|\mathbf{v}\|_{0,2}^{2} \mathrm{~d} t+\frac{1}{v} \int_{0}^{T}\|\mathbf{f}\|_{0,2}^{2} \mathrm{~d} t\right)
\end{aligned}
$$

which provides the second a priori estimate after using the first estimate to control the term involving $\|\mathbf{v}\|_{0,2}^{2}$.

\section{A UNIQUENESS RESULT}

Here we show that an additional regularity assumption is enough to obtain uniqueness.

\section{Theorem 4.1}

Let $\mathbf{v}_{1}, \mathbf{v}_{2} \in C_{\mathrm{w}}\left([0, T) ; H^{2}\left(\mathbb{R}^{3}\right)\right)$, be two solutions of Equations (4). Moreover, assume that $\mathbf{v}_{1} \in L^{1}\left(0, T ; W^{2,3}\left(\mathbb{R}^{3}\right)\right)$. Then $\mathbf{v}_{1}(t) \equiv \mathbf{v}_{2}(t)$ a.e. in $\mathbb{R}^{3}$ for all $t \geqslant 0$.

\section{Proof}

Subtracting Equation (4) $)_{1}$ written for the two solutions $\mathbf{v}_{1}$ and $\mathbf{v}_{2}$, multiplying the resulting equation by $\mathbf{w}=\mathbf{v}_{1}-\mathbf{v}_{2}$, and integrating over $\mathbb{R}^{3}$ provides the identity

$$
\begin{aligned}
\frac{1}{2} \frac{\mathrm{d}}{\mathrm{d} t} & \int_{\mathbb{R}^{3}}\left(|\mathbf{w}|^{2}+\alpha_{1}|\nabla \mathbf{w}|^{2}\right) \mathrm{d} x+v \int_{\mathbb{R}^{3}}|\nabla \mathbf{w}|^{2} \mathrm{~d} x \\
& +\frac{\beta}{2} \int_{\mathbb{R}^{3}}\left(\left|\mathbf{A}\left(\mathbf{v}_{1}\right)\right|^{2} \mathbf{A}\left(\mathbf{v}_{1}\right)-\left|\mathbf{A}\left(\mathbf{v}_{2}\right)\right|^{2} \mathbf{A}\left(\mathbf{v}_{2}\right)\right): \mathbf{A}(\mathbf{w}) \mathrm{d} x \\
= & -\int_{\mathbb{R}^{3}} \mathbf{w} \cdot \nabla \mathbf{v}_{1} \cdot \mathbf{w} \mathrm{d} x-\frac{\alpha_{1}}{2} \int_{\mathbb{R}^{3}} \mathbf{w} \cdot \nabla \mathbf{A}\left(\mathbf{v}_{1}\right): \mathbf{A}(\mathbf{w}) \mathrm{d} x \\
& -\alpha_{1} \int_{\mathbb{R}^{3}}\left(\mathbf{A}^{2}(\mathbf{w}): \mathbf{A}\left(\mathbf{v}_{1}\right)+\mathbf{A}\left(\mathbf{v}_{2}\right) \mathbf{A}(\mathbf{w}): \nabla \mathbf{w}\right) \mathrm{d} x \\
& -\frac{\alpha_{2}}{2} \int_{\mathbb{R}^{3}}\left(\mathbf{A}^{2}\left(\mathbf{v}_{1}\right)-\mathbf{A}^{2}\left(\mathbf{v}_{2}\right)\right): \mathbf{A}(\mathbf{w}) \mathrm{d} x
\end{aligned}
$$

It follows that

$$
\begin{aligned}
\frac{1}{2} \frac{\mathrm{d}}{\mathrm{d} t}( & \left.\|\mathbf{w}\|_{0,2}^{2}+\alpha_{1}\|\nabla \mathbf{w}\|_{0,2}^{2}\right)+v\|\nabla \mathbf{w}\|_{0,2}^{2}+\frac{\beta}{4} \int_{\mathbb{R}^{3}}\left(\left|\mathbf{A}\left(\mathbf{v}_{1}\right)\right|^{2}-\left|\mathbf{A}\left(\mathbf{v}_{2}\right)\right|^{2}\right)^{2} \mathrm{~d} x \\
& +\frac{\beta}{4} \int_{\mathbb{R}^{3}}|\mathbf{A}(\mathbf{w})|^{2}\left(\left|\mathbf{A}\left(\mathbf{v}_{1}\right)\right|^{2}+\left|\mathbf{A}\left(\mathbf{v}_{2}\right)\right|^{2}\right) \mathrm{d} x \\
\leqslant & c\left\|\nabla \mathbf{v}_{1}\right\|_{1,2}\|\mathbf{w}\|_{1,2}^{2}+c \alpha_{1}\|\mathbf{w}\|_{0,6}\left\|\nabla \mathbf{A}\left(\mathbf{v}_{1}\right)\right\|_{0,3}\|\mathbf{A}(\mathbf{w})\|_{0,2} \\
& +\frac{\beta}{8} \int_{\mathbb{R}^{3}}|\mathbf{A}(\mathbf{w})|^{2}\left(\left|\mathbf{A}\left(\mathbf{v}_{1}\right)\right|^{2}+\left|\mathbf{A}\left(\mathbf{v}_{2}\right)\right|^{2}\right) \mathrm{d} x+c \frac{\left(\left|\alpha_{1}\right|+\left|\alpha_{2}\right|\right)^{2}}{\beta}\|\nabla \mathbf{w}\|_{0,2}^{2}
\end{aligned}
$$


where we have used Hölder's and Young's inequalities, and Sobolev's embedding theorem. Hence, one obtains

$$
\begin{aligned}
\frac{1}{2} \frac{\mathrm{d}}{\mathrm{d} t}\left(\|\mathbf{w}\|_{0,2}^{2}+\alpha_{1}\|\nabla \mathbf{w}\|_{0,2}^{2}\right) \leqslant & c\left(\left\|\mathbf{v}_{1}\right\|_{2,2}+\max \left\{1, \alpha_{1}\right\}\left\|\nabla^{2} \mathbf{v}_{1}\right\|_{0,3}+\frac{\left(\left|\alpha_{1}\right|+\left|\alpha_{2}\right|\right)^{2}}{\alpha_{1} \beta}\right) \\
& \times\left(\|\mathbf{w}\|_{0,2}^{2}+\alpha_{1}\|\nabla \mathbf{w}\|_{0,2}^{2}\right)
\end{aligned}
$$

which, in view of Gronwall's inequality, yields the result.

\section{A BRIDGE BETWEEN EXISTENCE AND REGULARITY}

We are now ready to prove our main result providing a regularity class for the weak solution.

\section{Theorem 5.1}

Let $\mathbf{f} \in L^{2}\left(0, T ; H^{1}\left(\mathbb{R}^{3}\right)\right)$ and $\mathbf{v}_{0} \in H^{3}\left(\mathbb{R}^{3}\right)$. Moreover, assume that there exists a weak solution $\mathbf{v} \in C_{\mathrm{w}}\left([0, T) ; H^{2}\left(\mathbb{R}^{3}\right)\right)$ to problem (4) such that $\mathbf{v} \in L^{2}\left(0, T ; W^{2,3}\left(\mathbb{R}^{3}\right)\right)$. Then $\mathbf{v} \in L^{\infty}\left(0, T ; H^{3}\left(\mathbb{R}^{3}\right)\right)$, for all $T>0$.

Proof

The following calculations are formal but can be easily justified by a density argument. The idea is to test the equation

$$
\begin{aligned}
\partial_{t}\left(\mathbf{v}-\alpha_{1} \Delta \mathbf{v}\right)+(\mathbf{v} \cdot \nabla) \mathbf{v}-v \Delta \mathbf{v}-\beta \nabla \cdot\left(|\mathbf{A}(\mathbf{v})|^{2} \mathbf{A}(\mathbf{v})\right) \\
\quad=\mathbf{f}-\nabla p+\alpha_{2} \nabla \cdot \mathbf{A}^{2}(\mathbf{v})+\alpha_{1} \nabla \cdot\left(\mathbf{v} \cdot \nabla \mathbf{A}(\mathbf{v})+(\nabla \mathbf{v})^{\mathrm{T}} \mathbf{A}(\mathbf{v})+\mathbf{A}(\mathbf{v}) \nabla \mathbf{v}\right)
\end{aligned}
$$

with $\Delta^{2} \mathbf{v}$, perform integration by parts (typically twice), and estimate the resulting terms using the usual inequalities. First, one easily sees that

$$
\begin{aligned}
\int_{\mathbb{R}^{3}} \partial_{t} \mathbf{v} \cdot \Delta^{2} \mathbf{v} \mathrm{d} x & =\frac{1}{2} \frac{\mathrm{d}}{\mathrm{d} t} \int_{\mathbb{R}^{3}}\left|\nabla^{2} \mathbf{v}\right|^{2} \mathrm{~d} x \\
-\alpha_{1} \int_{\mathbb{R}^{3}} \partial_{t} \Delta \mathbf{v} \cdot \Delta^{2} \mathbf{v} \mathrm{d} x & =\alpha_{1} \int_{\mathbb{R}^{3}} \partial_{t} \nabla \mathbf{v}: \nabla \Delta^{2} \mathbf{v} \mathrm{d} x=\frac{\alpha_{1}}{2} \frac{\mathrm{d}}{\mathrm{d} t} \int_{\mathbb{R}^{3}}\left|\nabla^{3} \mathbf{v}\right|^{2} \mathrm{~d} x \\
-v \int_{\mathbb{R}^{3}} \Delta \mathbf{v} \cdot \Delta^{2} \mathbf{v} \mathrm{d} x & =v \int_{\mathbb{R}^{3}} \nabla \mathbf{v}: \nabla \Delta^{2} \mathbf{v} \mathrm{d} x=v \int_{\mathbb{R}^{3}}\left|\nabla^{3} \mathbf{v}\right|^{2} \mathrm{~d} x \\
\int_{\mathbb{R}^{3}} \mathbf{f} \cdot \Delta^{2} \mathbf{v} \mathrm{d} x & =-\int_{\mathbb{R}^{3}} \partial_{k} f_{i} \partial_{k} \Delta v_{i} \mathrm{~d} x \\
\int_{\mathbb{R}^{3}}(\mathbf{v} \cdot \nabla) \mathbf{v} \cdot \Delta^{2} \mathbf{v} \mathrm{d} x & =\int_{\mathbb{R}^{3}} \partial_{l} v_{j} \partial_{j} \partial_{k} v_{i} \partial_{k} \partial_{l} v_{i} \mathrm{~d} x-\int_{\mathbb{R}^{3}} \partial_{k} v_{j} \partial_{j} v_{i} \partial_{k} \partial_{l} \partial_{l} v_{i} \mathrm{~d} x:=I_{1}
\end{aligned}
$$


The next term is

$$
\begin{aligned}
-\beta \int_{\mathbb{R}^{3}} \nabla \cdot\left(|\mathbf{A}(\mathbf{v})|^{2} \mathbf{A}(\mathbf{v})\right) \cdot \Delta^{2} \mathbf{v} \mathrm{d} x= & \frac{\beta}{2} \int_{\mathbb{R}^{3}}|\mathbf{A}(\mathbf{v})|^{2} \mathbf{A}(\mathbf{v}) \cdot \mathbf{A}\left(\Delta^{2} \mathbf{v}\right) \mathrm{d} x \\
= & \frac{\beta}{2} \int_{\mathbb{R}^{3}} \partial_{k} \partial_{l}\left(|\mathbf{A}(\mathbf{v})|^{2} A_{i j}(\mathbf{v})\right) A_{i j}\left(\partial_{k} \partial_{l} \mathbf{v}\right) \mathrm{d} x \\
= & \frac{\beta}{2}\left\{\int_{\mathbb{R}^{3}}|\mathbf{A}(\mathbf{v})|^{2} A_{i j}\left(\partial_{k} \partial_{l} \mathbf{v}\right) A_{i j}\left(\partial_{k} \partial_{l} \mathbf{v}\right) \mathrm{d} x\right. \\
& +\int_{\mathbb{R}^{3}}\left(\partial_{k} \partial_{l}|\mathbf{A}(\mathbf{v})|^{2}\right) A_{i j}(\mathbf{v}) A_{i j}\left(\partial_{k} \partial_{l} \mathbf{v}\right) \mathrm{d} x \\
& \left.+2 \int_{\mathbb{R}^{3}}\left(\partial_{k}|\mathbf{A}(\mathbf{v})|^{2}\right) A_{i j}\left(\partial_{l} \mathbf{v}\right) A_{i j}\left(\partial_{k} \partial_{l} \mathbf{v}\right) \mathrm{d} x\right\}
\end{aligned}
$$

which can be rewritten in the form

$$
\frac{\beta}{2}\left\{\int_{\mathbb{R}^{3}}|\mathbf{A}(\mathbf{v})|^{2}\left|\nabla^{2} \mathbf{A}(\mathbf{v})\right|^{2} \mathrm{~d} x+\left.\left.\frac{1}{2} \int_{\mathbb{R}^{3}}\left|\nabla^{2}\right| \mathbf{A}(\mathbf{v})\right|^{2}\right|^{2} \mathrm{~d} x\right\}-I_{2}
$$

with

$$
I_{2}:=-\beta \int_{\mathbb{R}^{3}}\left(\partial_{k}|\mathbf{A}(\mathbf{v})|^{2}\right)\left(\partial_{k} \frac{|\mathbf{A}(\nabla \mathbf{v})|^{2}}{2}\right) \mathrm{d} x+\frac{\beta}{2} \int_{\mathbb{R}^{3}}\left(\partial_{k} \partial_{l}|\mathbf{A}(\mathbf{v})|^{2}\right) A_{i j}\left(\partial_{k} \mathbf{v}\right) A_{i j}\left(\partial_{l} \mathbf{v}\right) \mathrm{d} x
$$

The non-linear term multiplied by $\alpha_{2}$ takes the form

$$
\begin{aligned}
\alpha_{2} \int_{\mathbb{R}^{3}} \nabla \cdot \mathbf{A}^{2}(\mathbf{v}) \cdot \Delta^{2} \mathbf{v} \mathrm{d} x= & -\frac{\alpha_{2}}{2} \int_{\mathbb{R}^{3}} \mathbf{A}^{2}(\mathbf{v}): \mathbf{A}\left(\Delta^{2} \mathbf{v}\right) \mathrm{d} x \\
= & -\alpha_{2}\left\{\int_{\mathbb{R}^{3}} A_{i k}\left(\partial_{l} \partial_{m} \mathbf{v}\right) A_{k j}(\mathbf{v}) A_{i j}\left(\partial_{l} \partial_{m} \mathbf{v}\right) \mathrm{d} x\right. \\
& \left.+\int_{\mathbb{R}^{3}} A_{i k}\left(\partial_{l} \mathbf{v}\right) A_{k j}\left(\partial_{m} \mathbf{v}\right) A_{i j}\left(\partial_{l} \partial_{m} \mathbf{v}\right) \mathrm{d} x\right\}:=I_{3}
\end{aligned}
$$

and a similar reasoning shows that

$$
\begin{aligned}
\alpha_{1} \int_{\mathbb{R}^{3}} \nabla \cdot\left(\mathbf{v} \cdot \nabla \mathbf{A}(\mathbf{v})+(\nabla \mathbf{v})^{\mathrm{T}} \mathbf{A}(\mathbf{v})+\mathbf{A}(\mathbf{v}) \nabla \mathbf{v}\right) \cdot \Delta^{2} \mathbf{v} \mathrm{d} x \\
=-\frac{\alpha_{1}}{2}\left\{\int_{\mathbb{R}^{3}} \mathbf{v} \cdot \nabla \mathbf{A}(\mathbf{v}): \mathbf{A}\left(\Delta^{2} \mathbf{v}\right) \mathrm{d} x+\int_{\mathbb{R}^{3}} \mathbf{A}^{2}(\mathbf{v}): \mathbf{A}\left(\Delta^{2} \mathbf{v}\right) \mathrm{d} x\right\} \\
=-\frac{\alpha_{1}}{2}\left\{\int_{\mathbb{R}^{3}} A_{m l}(\mathbf{v}) \partial_{l} A_{i j}\left(\partial_{k} \mathbf{v}\right) A_{i j}\left(\partial_{k} \partial_{m} \mathbf{v}\right) \mathrm{d} x+\int_{\mathbb{R}^{3}} \partial_{k} \partial_{m} v_{l} \partial_{l} A_{i j}(\mathbf{v}) A_{i j}\left(\partial_{k} \partial_{m} \mathbf{v}\right) \mathrm{d} x\right\} \\
\quad-\alpha_{1}\left\{\int_{\mathbb{R}^{3}} A_{i k}\left(\partial_{l} \partial_{m} \mathbf{v}\right) A_{k j}(\mathbf{v}) A_{i j}\left(\partial_{l} \partial_{m} \mathbf{v}\right) \mathrm{d} x+\int_{\mathbb{R}^{3}} A_{i k}\left(\partial_{l} \mathbf{v}\right) A_{k j}\left(\partial_{m} \mathbf{v}\right) A_{i j}\left(\partial_{l} \partial_{m} \mathbf{v}\right) \mathrm{d} x\right\}:=I_{4}
\end{aligned}
$$


again because $\operatorname{div} \mathbf{v}=0$. This finally gives

$$
\begin{aligned}
& \frac{\mathrm{d}}{\mathrm{d} t}\left(\int_{\mathbb{R}^{3}}\left|\nabla^{2} \mathbf{v}\right|^{2} \mathrm{~d} x+\alpha_{1} \int_{\mathbb{R}^{3}}\left|\nabla^{3} \mathbf{v}\right|^{2} \mathrm{~d} x\right) x+2 v \int_{\mathbb{R}^{3}}\left|\nabla^{3} \mathbf{v}\right|^{2} \mathrm{~d} x \\
& \quad+\beta\left\{\int_{\mathbb{R}^{3}}|\mathbf{A}(\mathbf{v})|^{2}\left|\nabla^{2} \mathbf{A}(\mathbf{v})\right|^{2} \mathrm{~d} x+\left.\left.\frac{1}{2} \int_{\mathbb{R}^{3}}\left|\nabla^{2}\right| \mathbf{A}(\mathbf{v})\right|^{2}\right|^{2} \mathrm{~d} x\right\} \\
& \quad=-2 \int_{\mathbb{R}^{3}} \partial_{k} f_{i} \partial_{k} \Delta v_{i} \mathrm{~d} x-2 I_{1}+2 I_{2}+2 I_{3}+2 I_{4}
\end{aligned}
$$

The right-hand side in (12) can be bounded from above by

$$
\begin{aligned}
& 2\|\nabla \mathbf{f}\|_{0,2}\|\nabla \Delta \mathbf{v}\|_{0,2}+2 \beta\left\|\nabla|\mathbf{A}(\mathbf{v})|^{2}\right\|_{0,6}\|\nabla \mathbf{A}(\mathbf{v})\|_{0,3}\left\|\nabla^{2} \mathbf{A}(\mathbf{v})\right\|_{0,2} \\
& \quad+\beta\left\|\nabla^{2}|\mathbf{A}(\mathbf{v})|^{2}\right\|_{0,2}\|\nabla \mathbf{A}(\mathbf{v})\|_{0,3}\|\nabla \mathbf{A}(\mathbf{v})\|_{0,6}+2\|\nabla \mathbf{v}\|_{0,2}\left\|\nabla^{2} \mathbf{v}\right\|_{0,4}^{2} \\
& \quad+2\|\nabla \mathbf{v}\|_{0,4}^{2}\left\|\nabla^{3} \mathbf{v}\right\|_{0,2}+\left(2\left|\alpha_{1}+\alpha_{2}\right|+\alpha_{1}\right) \\
& \quad \cdot\left(\|\mid \mathbf{A}(\mathbf{v})\| \nabla^{2} \mathbf{A}(\mathbf{v})\|\|_{0,2}+2\left\|\nabla^{2} \mathbf{v}\right\|_{0,3}\|\nabla \mathbf{A}(\mathbf{v})\|_{0,6}\right)\left\|\nabla^{2} \mathbf{A}(\mathbf{v})\right\|_{0,2}
\end{aligned}
$$

where we have taken into account that $|\mathbf{A}(\mathbf{v})| \leqslant 2|\nabla \mathbf{v}|$. In view of Young's and Sobolev's inequalities, we get the estimates

$$
\begin{aligned}
& 2\|\nabla \mathbf{f}\|_{0,2}\|\nabla \Delta \mathbf{v}\|_{0,2} \leqslant \frac{1}{v}\|\nabla \mathbf{f}\|_{0,2}^{2}+v\left\|\nabla^{3} \mathbf{v}\right\|_{0,2}^{2} \\
& \beta\|\nabla \mathbf{A}(\mathbf{v})\|_{0,3}\left(2\left\|\nabla|\mathbf{A}(\mathbf{v})|^{2}\right\|_{0,6}\left\|\nabla^{2} \mathbf{A}(\mathbf{v})\right\|_{0,2}+\left\|\nabla^{2}|\mathbf{A}(\mathbf{v})|^{2}\right\|_{0,2}\|\nabla \mathbf{A}(\mathbf{v})\|_{0,6}\right) \\
& \quad \leqslant \frac{\beta}{4}\left\|\nabla^{2}|\mathbf{A}(\mathbf{v})|^{2}\right\|_{0,2}^{2}+c \beta\left\|\nabla^{2} \mathbf{v}\right\|_{0,3}^{2}\left\|\nabla^{3} \mathbf{v}\right\|_{0,2}^{2} \\
& \|\nabla \mathbf{v}\|_{0,2}\left\|\nabla^{2} \mathbf{v}\right\|_{0,4}^{2}+\|\nabla \mathbf{v}\|_{0,4}^{2}\left\|\nabla^{3} \mathbf{v}\right\|_{0,2} \leqslant c\left(1+\|\nabla \mathbf{v}\|_{1,2}^{2}\right)\left\|\nabla^{3} \mathbf{v}\right\|_{0,2}^{2}+c\|\nabla \mathbf{v}\|_{1,2}^{2} \\
& \left(2\left|\alpha_{1}+\alpha_{2}\right|+\alpha_{1}\right)\left(\|\mathbf{A}(\mathbf{v})\| \nabla^{2} \mathbf{A}(\mathbf{v}) \mid\left\|_{0,2}+2\right\| \nabla^{2} \mathbf{v}\left\|_{0,3}\right\| \nabla \mathbf{A}(\mathbf{v}) \|_{0,6}\right)\left\|\nabla^{2} \mathbf{A}(\mathbf{v})\right\|_{0,2} \\
& \quad \leqslant \frac{\beta}{2}\left\|\left|\mathbf{A}(\mathbf{v})\left\|\nabla^{2} \mathbf{A}(\mathbf{v}) \mid\right\|_{0,2}^{2}+c\left(v+\frac{\alpha_{1}^{2}}{\beta}+\left(\left|\alpha_{1}+\alpha_{2}\right|+\alpha_{1}\right)\left\|\nabla^{2} \mathbf{v}\right\|_{0,3}\right)\left\|\nabla^{3} \mathbf{v}\right\|_{0,2}^{2}\right.\right.
\end{aligned}
$$

where we have also recalled that $\left|\alpha_{1}+\alpha_{2}\right| \leqslant 24 \sqrt{v \beta}$ (cf. Equation (3)), and used the interpolation inequality

$$
\|\mathbf{v}\|_{0,4} \leqslant c\|\mathbf{v}\|_{0,2}^{1 / 4}\|\nabla \mathbf{v}\|_{0,2}^{3 / 4}
$$


In view of these estimates, we obtain, from (12), the inequality

$$
\begin{aligned}
& \frac{\mathrm{d}}{\mathrm{d} t}\left(\left\|\nabla^{2} \mathbf{v}\right\|_{0,2}^{2}+\alpha_{1}\left\|\nabla^{3} \mathbf{v}\right\|_{0,2}^{2}\right)+v\left\|\nabla^{3} \mathbf{v}\right\|_{0,2}^{2}+\left.\frac{\beta}{2}\|\mathbf{A}(\mathbf{v})\| \nabla^{2} \mathbf{A}(\mathbf{v})\left|\left\|_{0,2}^{2}+\frac{\beta}{4}\right\| \nabla^{2}\right| \mathbf{A}(\mathbf{v})\right|^{2} \|_{0,2}^{2} \\
& \quad \leqslant \frac{1}{v}\|\nabla \mathbf{f}\|_{0,2}^{2}+c\|\nabla \mathbf{v}\|_{1,2}^{2}+c\left(C\left(v, \alpha_{1}, \alpha_{2}, \beta\right)+\|\nabla \mathbf{v}\|_{1,2}^{2}+(\beta+1)\left\|\nabla^{2} \mathbf{v}\right\|_{0,3}^{2}\right)\left\|\nabla^{3} \mathbf{v}\right\|_{0,2}^{2}
\end{aligned}
$$

where $C\left(v, \alpha_{1}, \alpha_{2}, \beta\right)=c\left(1+v+\left(\alpha_{1}^{2} / \beta\right)+\left(\left|\alpha_{1}+\alpha_{2}\right|+\alpha_{1}\right)^{2}\right)$. From Gronwall's inequality it then follows that

$$
\begin{aligned}
\operatorname{ess} \sup _{0 \leqslant t \leqslant T}\left(\left\|\nabla^{2} \mathbf{v}\right\|_{0,2}^{2}+\alpha_{1}\left\|\nabla^{3} \mathbf{v}\right\|_{0,2}^{2}\right)+v \int_{0}^{\mathrm{T}}\left\|\nabla^{3} \mathbf{v}\right\|_{0,2}^{2} \mathrm{~d} t \\
\leqslant \exp \left\{C\left(v, \alpha_{1}, \alpha_{2}, \beta\right) T+\int_{0}^{\mathrm{T}}\|\nabla \mathbf{v}\|_{1,2}^{2} \mathrm{~d} t+(\beta+1) \int_{0}^{\mathrm{T}}\left\|\nabla^{2} \mathbf{v}\right\|_{0,3}^{2} \mathrm{~d} t\right\} \\
\quad\left(\left\|\nabla^{2} \mathbf{v}_{0}\right\|_{0,2}^{2}+\alpha_{1}\left\|\nabla^{3} \mathbf{v}_{0}\right\|_{0,2}^{2}+\frac{1}{v} \int_{0}^{\mathrm{T}}\|\nabla \mathbf{f}\|_{0,2}^{2} \mathrm{~d} s+c \int_{0}^{\mathrm{T}}\|\nabla \mathbf{v}\|_{1,2}^{2} \mathrm{~d} t\right)
\end{aligned}
$$

which concludes the proof in view of the first two a priori estimates (6) and (9), and the regularity assumption $\mathbf{v} \in L^{2}\left(0, T ; W^{2,3}\left(\mathbb{R}^{3}\right)\right)$.

We can obtain further regularity by testing Equation (4) $)_{1}$ with $-\Delta^{3} \mathbf{v}$ and performing again some integrations by parts. We obtain the inequality

$$
\begin{aligned}
& \frac{\mathrm{d}}{\mathrm{d} t}\left(\left\|\nabla^{3} \mathbf{v}\right\|_{0,2}^{2}+\alpha_{1}\left\|\nabla^{4} \mathbf{v}\right\|_{0,2}^{2}\right)+2 v\left\|\nabla^{4} \mathbf{v}\right\|_{0,2}^{2}+\beta \int_{\mathbb{R}^{3}}|\mathbf{A}(\mathbf{v})|^{2}\left|\nabla^{3} \mathbf{A}(\mathbf{v})\right|^{2} \mathrm{~d} x+\left.\left.\frac{\beta}{2} \int_{\mathbb{R}^{3}}\left|\nabla^{3}\right| \mathbf{A}(\mathbf{v})\right|^{2}\right|^{2} \mathrm{~d} x \\
& \leqslant c \int_{\mathbb{R}^{3}}\left(|\nabla \mathbf{v}|\left|\nabla^{3} \mathbf{v}\right|^{2}+\left|\nabla^{2} \mathbf{v}\right|^{2}\left|\nabla^{3} \mathbf{v}\right|\right) \mathrm{d} x+2\left|\left(\nabla^{2} \mathbf{f}, \nabla^{4} \mathbf{v}\right)\right| \\
& \quad+C\left(\alpha_{1}, \alpha_{2}\right) \int_{\mathbb{R}^{3}}\left(|\mathbf{A}(\mathbf{v})|\left|\nabla^{3} \mathbf{A}(\mathbf{v})\right|^{2}+\left|\nabla^{2} \mathbf{v}\right|\left|\nabla^{3} \mathbf{v}\right|\left|\nabla^{4} \mathbf{v}\right|\right) \mathrm{d} x \\
& \quad+c(\beta) \int_{\mathbb{R}^{3}}|\mathbf{A}(\mathbf{v})||\nabla \mathbf{A}(\mathbf{v})|\left|\nabla^{2} \mathbf{A}(\mathbf{v})\right|\left|\nabla^{3} \mathbf{A}(\mathbf{v})\right|+|\nabla \mathbf{A}(\mathbf{v})|^{2}\left|\nabla^{2} \mathbf{A}(\mathbf{v})\right|^{2} \mathrm{~d} x
\end{aligned}
$$

from which one easily concludes, using the previous estimates, that

$$
\mathbf{v} \in L^{\infty}\left(0, T ; H^{4}\left(\mathbb{R}^{3}\right)\right) \text { for all } T>0
$$

assuming additional hypotheses on the initial datum, $\mathbf{v}_{0} \in H^{4}\left(\mathbb{R}^{3}\right)$, and on the forcing term, $\mathbf{f} \in L^{2}\left(0, T ; H^{2}\left(\mathbb{R}^{3}\right)\right)$.

\section{ACKNOWLEDGEMENTS}

This research was largely supported by ICCTI/DAAD, through an INIDA Programme. The research of J. M. Urbano was also supported by CMUC/FCT and the research of J. H. Videman by CEMAT/FCT. 


\section{REFERENCES}

1. Agranovich YY, Sobolevskii PE. Motion of nonlinear visco-elastic fluid. Nonlinear Analysis 1998; 32:755-760.

2. Bresch D, Lemoine J. On the existence of solutions for non-stationary third-grade fluids. International Journal of Non-Linear Mechanics 1999; 34:485-498.

3. Cioranescu D, Girault V. Weak and classical solutions of a family of second grade fluids. International Journal of Non-Linear Mechanics 1997; 32:317-335.

4. Cioranescu D, Ouazar EH. Existence and Uniqueness for Fluids of Second Grade. Pitman Research Notes in Mathematics, vol. 109. Collége de France Seminars, Pitman: London, 1984; 178-197.

5. Fernández-Cara E, Guillén F, Ortega R. Some theoretical results concerning non-Newtonian fluids of the Oldroyd kind. Annali della Scuola Normale Superiore di Pisa. Classe di Scienze. Serie IV 1998; 26:1-29.

6. Galdi GP, Grobbelaar M, Sauer N. Existence and uniqueness of classical solutions of the equations of motion for second-grade fluids. Archive for Rational Mechanics and Analysis 1993; 124:221-237.

7. Galdi GP, Sequeira A. Further existence results for classical solutions of the equations of a second-grade fluid. Archive for Rational Mechanics and Analysis 1994; 128:297-312.

8. Guillopé C, Saut JC. Existence results for the flow of viscoelastic fluids with a differential constitutive law. Nonlinear Analysis 1990; 15:849-869.

9. Sequeira A, Videman JH. Global existence of classical solutions for the equations of third grade fluids. Journal of Mathematical Physical Sciences 1995; 29:47-69.

10. Busuioc V, Iftimie D. Global existence and uniqueness of solutions for the equations of third grade fluids. International Journal of Non-Linear Mechanics 2004; 39:1-12.

11. Ladyzhenskaya OA. New equations for the description of motion of viscous incompressible fluids and solvability in the large of boundary value problems for them. Trudy Matematicheskogo Instituta Imeni V.A. Steklova 1967; 102:95-118.

12. Rivlin RS, Ericksen JL. Stress-deformation relations for isotropic materials. Journal of Rational Mechanics and Analysis $1955 ; 4: 323-425$.

13. Fosdick RL, Rajagopal KR. Thermodynamics and stability of fluids of third grade. Proceedings of the Royal Society of London, Series A 1980; 339:351-377. 